\title{
Unmanned aerial vehicles optimal airtime estimation for energy aware deployment in loT-enabled fifth generation cellular networks
}

\author{
Saqib Majeed ${ }^{1}$, Adnan Sohail ${ }^{1}$, Kashif Naseer Qureshi² ${ }^{*}$ (D), Arvind Kumar ${ }^{3}$, Saleem lqbal ${ }^{4}$ and Jaime Lloret $^{5}$
}

\author{
*Correspondence: \\ kashifnq@gmail.com \\ 2 Department of Computer \\ Science, Bahria University, \\ Islamabad, Pakistan \\ Full list of author information \\ is available at the end of the \\ article
}

\begin{abstract}
Cellular networks based on new generation standards are the major enabler for Internet of things (IoT) communication. Narrowband-IoT and Long Term Evolution for Machines are the newest wide area network-based cellular technologies for loT applications. The deployment of unmanned aerial vehicles (UAVs) has gained the popularity in cellular networks by using temporary ubiquitous coverage in the areas where the infrastructure-based networks are either not available or have vanished due to some disasters. The major challenge in such networks is the efficient UAVs deployment that covers maximum users and area with the minimum number of UAVs. The performance and sustainability of UAVs is largely dependent upon the available residual energy especially in mission planning. Although energy harvesting techniques and efficient storage units are available, but these have their own constraints and the limited onboard energy still severely hinders the practical realization of UAVs. This paper employs neglected parameters of UAVs energy consumption in order to get actual status of available energy and proposed a solution that more accurately estimates the UAVs operational airtime. The proposed model is evaluated in test bed and simulation environment where the results show the consideration of such explicit usage parameters achieves significant improvement in airtime estimation.
\end{abstract}

Keywords: Energy aware, UAV, UE, Dynamic deployment, Communicational energy, Energy efficiency

\section{Introduction}

The cellular coverage using unmanned aerial vehicles (UAVs) is gaining attention from research community and telecom industry with the rapid deployment of Internet of things (IoTs). For collection/dissemination of data, IoTs-based cellular technologies are being provided [1]. For provision of services in wider coverage area, the cellular networks are augmented with UAVs. UAVs are the trustable solution for improving of the efficiency, enhance throughout, cost and boosting capacity [2]. These UAVs are temporarily deployed in the air to cover an area where the user demand is increased or in the case of disasters or to provide connectivity in areas where permanent infrastructure is not currently possible. This also includes areas where physical infrastructure is available, but the user density is very high (event, stadium, etc.). The

OThe Author(s) 2020. Open Access This article is licensed under a Creative Commons Attribution 4.0 International License, which permits use, sharing, adaptation, distribution and reproduction in any medium or format, as long as you give appropriate credit to the original author(s) and the source, provide a link to the Creative Commons licence, and indicate if changes were made. The images or other third party material in this article are included in the article's Creative Commons licence, unless indicated otherwise in a credit line to the material. If material is not included in the article's Creative Commons licence and your intended use is not permitted by statutory regulation or exceeds the permitted use, you will need to obtain permission directly from the copyright holder. To view a copy of this licence, visit http:// creativecommons.org/licenses/by/4.0/. 
developments of UAVs not only provide the solution but also provide the load balancing, and easily cover the maximum demanding area [3]. For this purpose, an efficient approach and mechanism is required.

Previously, Cell on Wheel (COW) is used as a temporary solution in which a vehicle was designed to carry a mobile microcellular base station [4]. This solution was developed in conjunction with the Telstra Next Generation deployment plan to extend the coverage area to cover any event or emergency [5]. There are few situations in which the cow could fail, for example in areas of natural disaster where the road infrastructure necessary for displacement is not available. The basic reason for UAVs as a favorable solution for a wide range of neighborhood applications is the ability of its free and independent movement, to any hard to reach areas [6,7]. These UAVs are furnished with the base station (BS) hardware, and these act as a flying BS, creating an attractive alternative to predictable roof or pole attached base stations. In radio communication, the BS is a wireless communication station mounted at a fixed location and used to connect as part of wireless telephone scheme. The BS relays the conversation, message and data to base stations in other cells by the wireless, cable communication or through a cable network or through a combination of wireless and cables.

The placement of these UAV-based BS in a microcell network in order to get optimal coverage area where the user are not stationary is a challenging task $[8,9]$. Providing best placement while keeping the number of UAVs optimal is hard to achieve. Multiple solutions for this problem are discussed in the literature, where majority have focused on provision of the energy efficient solutions for placement of UAVs. Among them, very less attention has been paid toward energy aware solutions and additionally; such solutions have only considered implicit utilization of energy to estimate the available energy [10]. However, the explicit usage of UAV energy has not been addressed yet, which also has severe effect on the UAV survivability.

The main contribution of this paper is twofold:

- Identification of UAVs exergy explicit usage parameters

- To provide a solution that more accurately estimates the UAVs operational airtime

The rest of this paper is organized as follows. Section 2 presents the related work. The methodology describes in Sect. 3. Prototyping details are explained in Sect. 4. The experiment results are present in Sect. 5. Section 4 includes the conclusion and future directions of our research.

\section{Related work}

The UAV base stations (UAV-BS) enhance network coverage and area capacity by moving supply toward demand when required [11, 12]. However, deployment of such UAVBS can face certain restrictions that need to be considered while designing a solution. The major concern is the lifetime of a UAV for which it could remain in operation. The most important factor affecting the UAVs lifetime is energy source. Following are some of the recent papers related to UAV-BS in cellular networks that focus on energy aware deployments. Thus, it is the duration for which the ground users are getting the services. 
The prolonged lifetime in UAV-based network is normally achieved by designing energy efficient solutions [13-16]. Wang et al. [17] suggested in their work set of rules for UAV base stations that predicted an energy-efficient placement so that should serve users by minimal transmit energy. Also, the most appropriate placement of the UAVBS was presented in their work by decoupling the deployment problem in horizontal and vertical dimension determined the most desirable UAV position that minimizes the transmission power by performing the simulations for hotspot and non-hotspot scenarios.

Chen, et al. [14] describe a battery-operated version of the UAV power consumption model which is then applied to a situation of UAV flight. The UAV consumes less electricity while it travels at excessive horizontal speed at some stage in the task, because low speed is not really greatest for the UAV for the energy scenario. The cause is that the hovering power is stable ultimately after the flying time. Cabreira, et al. [18] write in the paper that in case of using UAV automobile dynamics, turning angle and optimal speed must be considered to minimize electricity intake. Their proposed algorithms mainly involved about energy intake considering the mission and electricity constraints of the UAVs. Thus, improving velocity in straight parts of the path leads to energy consumption minimization. In the paper, the path divided into forty-five small elements (straights and curves) for every straight a part of the coverage it became taken into consideration: the acceleration, the steady speed flight and the deceleration. By using the equal velocity of 8 . zero $\mathrm{m} / \mathrm{s}$ for long elements and the reduction of $45 \%$ in velocity before turns referred to as entrance speed of UAV by using a battery of value 46, $681 \mathrm{~J}$.

Another paper [15] proposed a scheme which presents the UAV offloading (air-offloading and ground-offloading) approaches. In the air-offloading approach, a UAV can offload its computing responsibilities to nearby UAVs that have to be had computing and electricity sources. The floor-offloading technique enables the project offloading carrier to an edge cloud server that is related to floor stations. This hybrid offloading scheme comprises three major modules to lengthen the life of UAVs by way of saving resources through task offloading process. Furthermore, this scheme efficiently reduces the project blocking probability and the cease-to-quit latency of managing a computing mission.

Fotouhi, et al. [16] provided option to improve communique power performance is to develop optimum transmission schedule of UAVs, mainly while UAVs are flying in a predetermined trajectory fixed-wing UAVs can movement over the air, which makes them substantially more energy effective and capable of carry heavy payload. Small commercial UAVs typically have a flight time of 20-30 min, while some big UAVs can last for hours. Researchers in this study focused on two types of energy saving reducing communication energy minimize the transmission power energy efficiency is to develop most desirable transmission time table of UAVs, especially while UAVs are flying in a predetermined trajectory. To reduce mechanical cost an energy intake model is needed. However, changing the height might reduce the performance of UAVs.

However, in these solutions the focus is on a less energy usage in terms of computational power and does not focus on transmission requirement or estimation that for how long the UAV remains present in the air. There are some solutions that optimize the energy consumption by minimizing the transmission power $[19,20]$. The decision regarding the use of energy consumption has been implemented both as centralized as 
well as distributed approach. In [21], the author has proposed an approach in which the UAV report the statistics to the base station and the BS proposes a scheduling model that minimizes the UAV energy consumption. The base station in its scheduling informs the UAV about the time slot and power information. A similar centralized entity-based solution has also been proposed that is dependent upon cluster heads [22]. These cluster heads (Terrestrial nodes) are in direct communication with the UAV and ground users are connected with the cluster heads. But these types of solutions have their own limitations.

However, these solutions are also dependent on the estimated energy that accordingly adjusts the transmission power, this way; the UAV coverage is also affected. A third side of energy, the mechanical energy, has also been discussed by some researchers [23]. These solutions reduce mechanical energy of UAVs and are dependent upon multirotor, fixed-wing and hybrid fixed/rotary UAVs. Similarly, in [24], the authors isolated the two power consumptions into static and dynamic, the static the fixed consumption of the UAV and dynamic is dependent upon the load either low traffic load or high traffic load. In another study [25], the authors suggested a dynamic planning that is also dependent upon traffic intensity. They proposed to have different planning for day and nights, as in night the active users are less as compared to day timings and accordingly they plan the placement of UAV.

In recent past, multiple studies have been made in the domain of UAV placement as it could provide a better alternative for temporary cell construction in the uncomplimentary cell sites. The parameters that influence the placement of UAV are key factors that can severely affect the network performance. The lifetime of a UAV is greatly dependent upon the energy available. From the literature, six types of energy consumption parameters have been extracted. Energy spent on (a) horizontal movement, (b) vertical movement, (c) hovering, (d) processing, (e) backbone connectivity/communication and (f) providing front haul/ access to users. However, all these six parameters have not been given full consideration for energy aware solutions, which provide the estimated time of UAV for which it can remain in the air. This estimation greatly helps in UAVs placement/ replacement planning.

Authors in [17] proposed an energy-efficient placement algorithm by decoupling the drone deployment in horizontal and vertical locations, by using minimum required transmit power. They perform simulation on hotspot and non-hotspot scenarios, and the numerical results show the linear association between the minimum horizontal location and optimal altitude. They first find the optimal horizontal position of the Drone base station (DBS). The average path loss against the altitudes for various horizontal distances between the edge users and the drone, there lies a point of minimum value of average path loss of the edge users and the relevant drone altitude. Furthermore, by decreasing the horizontal distance of the edge users from the DBS, the minimum average path loss of the edge and the altitude decrease. Once DBS obtains the minimum horizontal distance, corresponding optimal drone altitude is calculated. The results show the power saving in the urban, suburban and dense urban environments optimal altitude.

In [26], the researcher develops the photovoltaic power management system (PPMS) which manages power from photovoltaic modules and a battery pack for multirotor UAV power. They use the state of charge concepts grounded on extended Kalman filter 
(EKF) and complementary filter (CF) for estimation of flight time. It also calculates the possible flight time during hovering flight mode and hint of the remaining energy of the battery pack by using the slope of the state of charge graph. During takeoff, hovering and landing flight modes patterns are estimated in these three modes of UAVs and the mean value is calculated. There were three main power connectors connected to the photovoltaic modules, battery pack and UAV. Voltage and the current of all three main power connectors were monitored. During low sunlight, battery pack mostly deliveries the required drone power. During daytime, photovoltaic modules deliver the power required from the drone. They keep track of the voltage and current data of the photovoltaic modules, battery pack and UAV, progress time and battery pack temperature. According to the results, estimated flight time increased up to $54.14 \mathrm{~min}$ at 11:00 a.m. and decreased down to $6.70 \mathrm{~min}$ at 18:00 p.m. The results also indicated that if there were no clouds covering the sun, the UAV could fly for about an hour at around noon which was much higher than the flight time of the traditional multirotor UAV. The future work is to improve the current version of the PPMS.

\section{Methodology-UAV airtime estimation for energy aware deployment in loT}

\subsection{UAV system model}

The airtime estimation is dependent upon three parameters: (a) the available capacity of the battery mounted on the UAV, (b) its discharge limit and (c) the average amp draw. In almost all of the previous research work and practical deployments, these three parameters have been considered. For this, the following equation is used:

$$
\mathrm{AT}=C_{\mathrm{B}} D_{\mathrm{L}} / \mathrm{AA} \mathrm{D}_{\mathrm{D}}
$$

where AT is the airtime, $C_{\mathrm{B}}$ is the battery capacity and $D_{\mathrm{L}}$ is the allowed discharge limit, in normal practice, the discharge limit is set to $80 \%$, whereas the $A A_{D}$ is the Average Amp Draw which is calculated in amperes using following Eq. 2:

$$
\mathrm{AA}_{\mathrm{D}}={ }_{\mathrm{C}} \frac{P_{\mathrm{W}}}{\Delta V}
$$

where $W_{\mathrm{C}}$ represents the weight carried, which represents the total weight of the UAV including the equipment/battery that will be carried by the UAV; usually, it is measured in kilograms. The $P_{\mathrm{W}}$ is the power required to carry one kilogram of weight, normally expressed in watts per kilogram. The $\Delta V$ is the battery voltage, expressed in volts. However, the estimated airtime may differ from the real airtime such as in extreme wind cases; the airtime may decrease up to $50 \%$.

Such estimation normally gives a generic value. The actual estimation is also influenced by the task being performed by the UAV. In the proposed work, the UAV is carrying a base station as shown in modular form in Fig. 1. This base station has connectivity modules for communication with backbone and frontend module for providing access services to the ground users as shown in Fig. 2. Both of these modules are also dependent upon the same battery mounted on UAV for its flying (implicit usage). The details of flow represented in Fig. 2 are calculated by using Eqs. 3 and 4. The flying requires rotation of motors; hence, such usage has also been termed as mechanical energy in the literature [27]. 


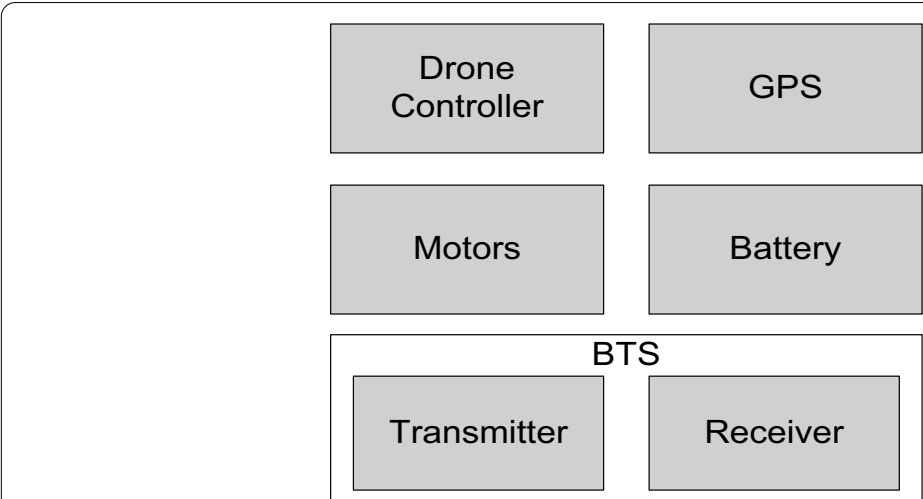

Fig. 1 UAV-BS core components

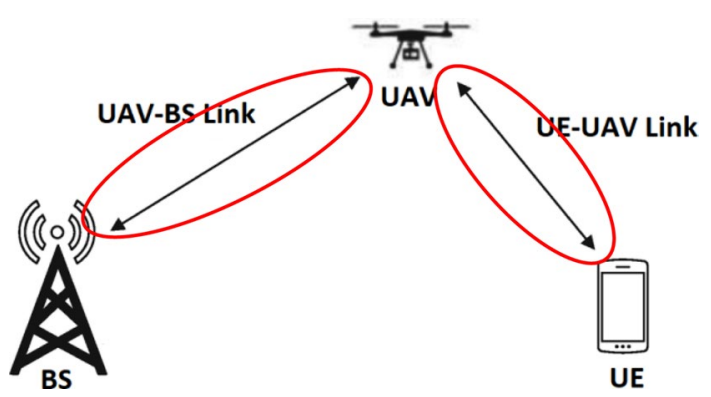

Fig. 2 Explicit energy usage for UAV

In the proposed work, the explicit usage (backend and frontend communication) is a key factor in UAV-BS scenario; therefore, it is given the due consideration. However, the communication energy overhead in other UAV deployments is considered negligible [27]. The main task performed by the UAV-BS is communication; hence, communication energy is incorporated as depicted in Fig. 3. Here the bidirectional links, highlighted in red, represents the uplink and downlink from/toward UAV from user equipment (UE) and base station (BS). Accordingly, Eq. 3 is extended as follows:

$$
\mathrm{AT}=C_{\mathrm{B}} D_{\mathrm{L}} /\left(\mathrm{AA} \mathrm{D}+E_{\mathrm{U}}\right)
$$

The $E_{\mathrm{U}}$ in the above equation represents the explicit usage factor. The average energy consumptions of a base transceiver station (BTS) is associated with the communication technology being used, as depicted in the literature while comparing Global System Mobile (GSM) and Universal Mobile Telephone Services (UMTS), it was noted that the GSM considerably have higher energy consumption than the UMTS technology [28]. Similarly, multiple energy consumption analyses have been performed for various communication technologies such as for Bluetooth, Wi-Fi and Cellular Networks [29, 30].

\subsection{Energy aware deployment scenarios}

The Explicit Usage factor is dependent upon the user location, density and the UAV coverage radius [16]. The user density is normally divided into three categories: (a) suburban 


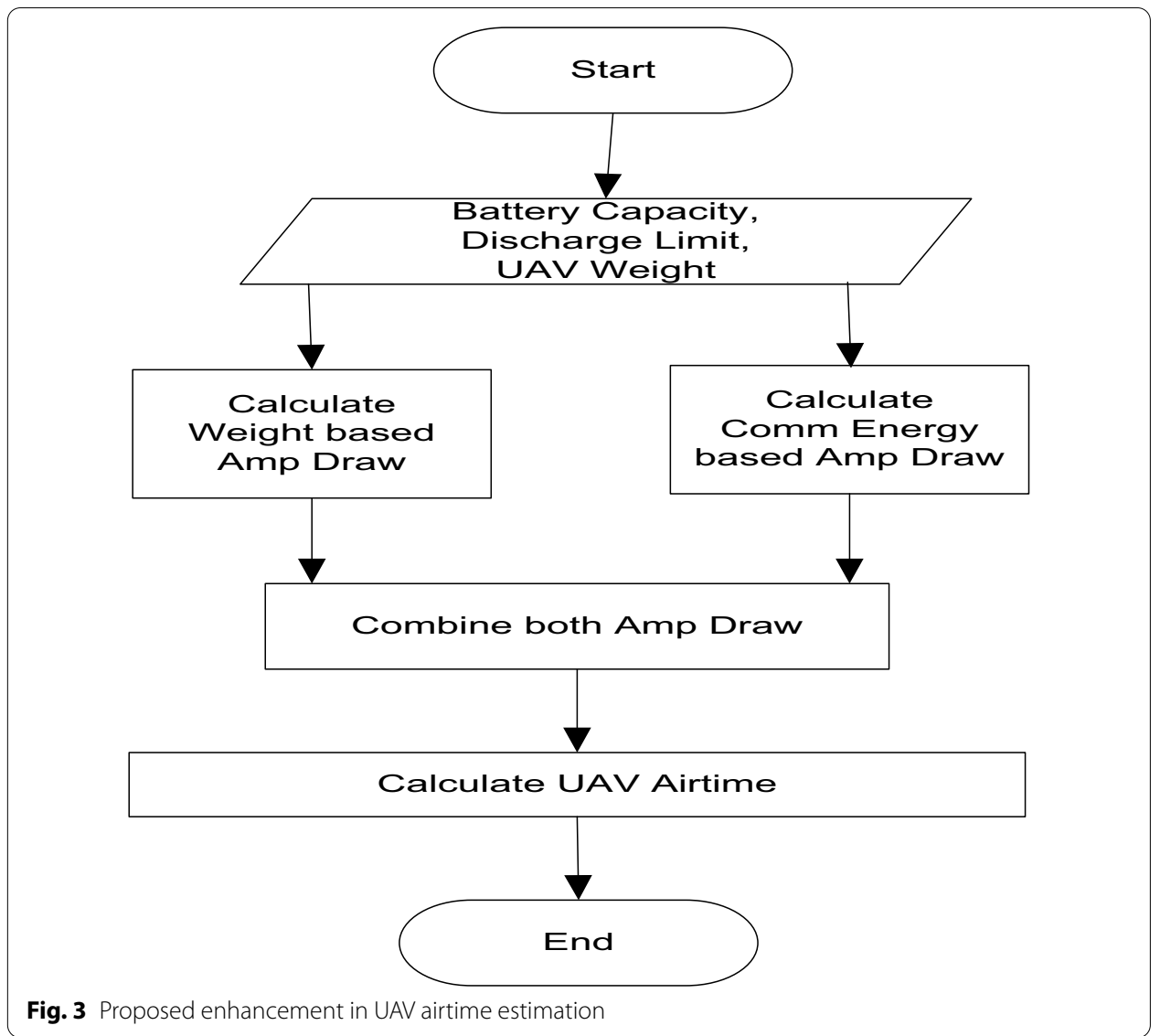

(b) urban and (c) dense urban [17]. Healthcare is the prominent use case for urban drone deployment [31,32]. The users on the boundary of the coverage area are the users with the maximum horizontal distance from the UAV, normally referred edge users. While calculating the UAV coverage radius, such edge users have also been considered along with the UAV altitude [17]. The transmission power required by a UAV considering the user density and the UAV coverage area is calculated by [33] and is depicted in Table 1 . Here, secure data transmission is a priority for this study considering healthcare application of drones [34]. It is observed that having greater number of cells (more UAVs) requires a transmission power that is not having severe effect but when there are less numbers of cells, a quite high transmit power of $(\geq 53.9 \mathrm{~W})$ is required. This effect is mathematically incorporated using the following equation.

$$
E_{\mathrm{U}}=\frac{P_{\mathrm{T}}}{\Delta V}
$$

where $P_{\mathrm{T}}$ is the transmission power required by a UAV in order to cover a microcell and $V$ is the battery voltage.

An example for UAV airtime calculation has been performed in [35]. Let us mathematically analyze both of our cases of with and without Explicit Usage factor using the same example, the specifications and their values used for the example are given in Table 2.

Scenario 1 (a): Without explicit usage 
Table $1 P_{\top}$ requirement to cover active users in each circle cell [31]

\begin{tabular}{|c|c|c|c|c|c|}
\hline \multicolumn{3}{|c|}{ (a) Rectangular region divided into $\mathrm{n}$ cells } & \multicolumn{3}{|c|}{ (b) Circular region divided into $n$ cells } \\
\hline Cells $(n)$ & Circular radius & $\begin{array}{l}\text { UAV transmit } \\
\text { power (W) }\end{array}$ & Cells $(n)$ & Circular radius & UAV transmit power (W) \\
\hline 10 & $493 \mathrm{~m}$ & Very high & 8 & 340 & 53,564 very high \\
\hline 15 & $402 \mathrm{~m}$ & Very high & 9 & 311 & 267.1 very high \\
\hline 18 & $368 \mathrm{~m}$ & 53.9 high & 10 & 295 & 17.43 \\
\hline 19 & $357 m$ & 11.95 & 11 & 287 & 4.29 \\
\hline 20 & $351 \mathrm{~m}$ & 6.088 & 12 & 279 & 1.63 \\
\hline 21 & $345 \mathrm{~m}$ & 3.306 & 13 & 266 & 0.166 \\
\hline 22 & $341 \mathrm{~m}$ & 1.59 & 14 & 260 & 0.0874 \\
\hline 23 & $339 \mathrm{~m}$ & 1.013 & 15 & 249 & 0.0199 \\
\hline 24 & $334 \mathrm{~m}$ & 0.847 & 16 & 244 & $9.80 \times 10^{-3}$ \\
\hline 25 & $331 \mathrm{~m}$ & 0.435 & 17 & 235 & $2.40 \times 10^{-3}$ \\
\hline
\end{tabular}

Table 2 UAV airtime calculation specifications

\begin{tabular}{ll}
\hline Specifications & Values \\
\hline Battery capacity & $8.8 \mathrm{Ah}$ \\
Discharge limit & $80 \%$ \\
Battery voltage (VOL) & $36 \mathrm{~V}$ \\
UAV weight (with LiPo battery) & $2.5 \mathrm{~kg}$ \\
Power (PWR W/kg) & $170 \mathrm{~W} / \mathrm{kg}$ \\
\hline
\end{tabular}

For calculating UAV airtime (AT) without any explicit usage, the following steps are followed:

- Calculating Average Amp Draw using Eq. 2

- Calculating UAV airtime using Eq. 1

Scenario 1 (b): Without Explicit Usage (with increasing UAV height)

The flying height of the UAV has its influence not only on the coverage of the base station and user density but also results in a higher power usage. Here, link quality consideration can also be a significant point focusing on the highly mobility drone networking environment [36]. Increasing the altitude by $15 \mathrm{~m}$ results in consumption of an additional power of $0.5 \mathrm{~A}$. If this is also included into the airtime calculation, Eq. 2 is extended in order to cover Amps for the height factor $\left(A_{\mathrm{HF}}\right)$ as represented in Eq. 7.

$$
\mathrm{AA}_{\mathrm{D}}={ }_{C}\left(\frac{P_{\mathrm{W}}}{V}+A_{\mathrm{HF}}\right)
$$

Now following steps are followed for calculating UAV airtime (AT) without any explicit usage but considering height factor.

- Calculating $\mathrm{AA}_{\mathrm{D}}$ using Eq. 5

- Calculating UAV airtime using Eq. 1 
Scenario 2: With explicit usage

- Calculating the explicit usage ( $\left.E_{\mathrm{U}}\right)$ factor using Eq. 4

- Calculating $\mathrm{AA}_{D}$ using Eq. 2

- Calculating UAV airtime (AT using Eq. 3

For the proof of concept, consider the following example cases:

Case I: Transmission Power $\left(P_{\mathrm{T}}\right)=53.9 \mathrm{~W}$ (based on Table 1a).

Case II: Transmission Power $\left(P_{\mathrm{T}}\right)=267.1 \mathrm{~W}$ (based on Table $\left.1 \mathrm{~b}\right)$.

In scenario 2, the UAV hovers for $30 \mathrm{~min}$ and $21.6 \mathrm{~min}$, respectively, for cases I and II. If compared with scenario I (without Explicit Usage Factor), the calculations show that the airtime of 36 min difference makes a lot of impact while planning deployment of UAVs.

\subsection{Drone prototype development}

A UAV prototype is indigenously developed as depicted in Fig. 4. For backhaul and front haul communication, a local wireless service provider's dongle (5G) was used that also provides IEEE $802.11(\mathrm{~b} / \mathrm{g} / \mathrm{n}$ )-based access network as shown in Fig. 5. This supports up to ten connections simultaneously over Wi-Fi and have 2,380 $\mathrm{mAh}$ battery for up to $5 \mathrm{~h}$ of constant usage. To accommodate the maximum number of users, an additional wireless router is plugged. The detailed specifications are given in Table 3.

The UAV profiles were obtained for transmission requirement at different heights and analyzed accordingly.

\section{Results and discussion}

The UAV altitude affects the energy from two perspectives: (a) the energy required by motors to increase the UAV altitude and (b) with higher altitude the transmission power also increases in order to cover the required area on ground. In this research, takeoff, landing and moving vertical or horizontal while changing the altitude and increasing the coverage radius have not considered. But because we are working on $80 \%$ usage of UAV so its rational to ignore such parameters in order to focus the transmission impact on

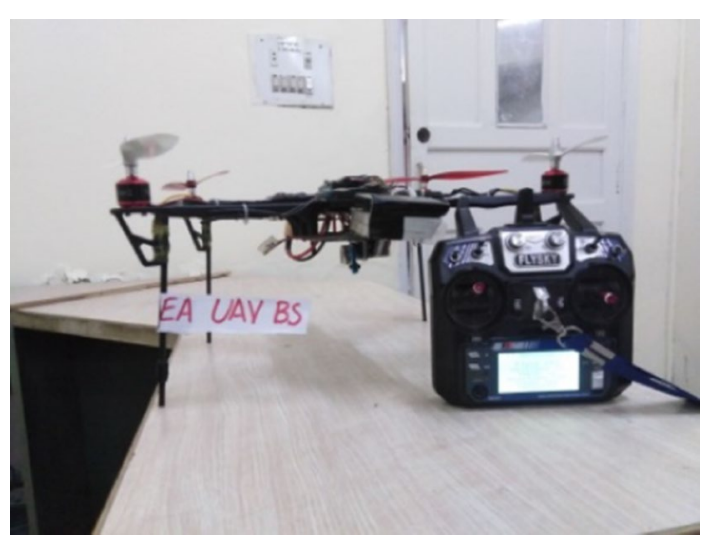

Fig. 4 Side view-UAV prototype 


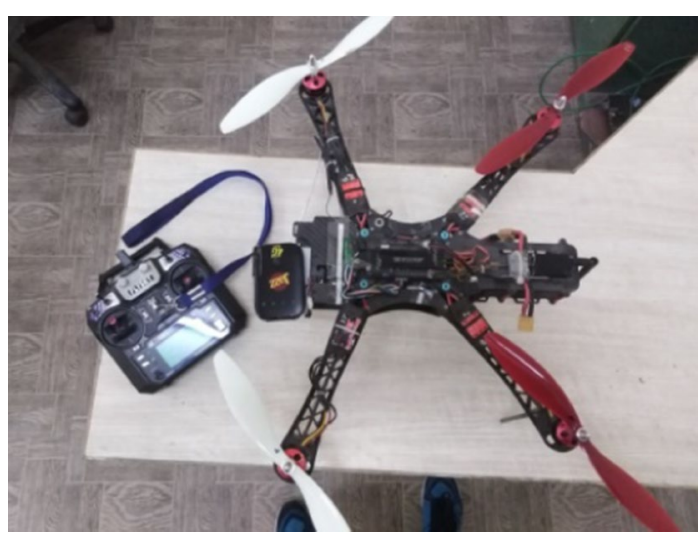

Fig. 5 Upper view-UAV prototype

Table 3 UAV specifications

\begin{tabular}{ll}
\hline Module & Specification \\
\hline Flight controller & APM 2.8 \\
Device aerial vehicle & Servo Parabolic Switch 13.76 X100 \\
Power distribution & Board XT60 For Quadcopter UAV 3.94 X100 \\
Microcontroller & Raspberry Pi kit and TF card 16 GB 57.50 X100 \\
Frame kit & 500 mm Quadcopter $30.00 \times 100$ \\
Dongle & 4G Hotspot device \\
\hline
\end{tabular}

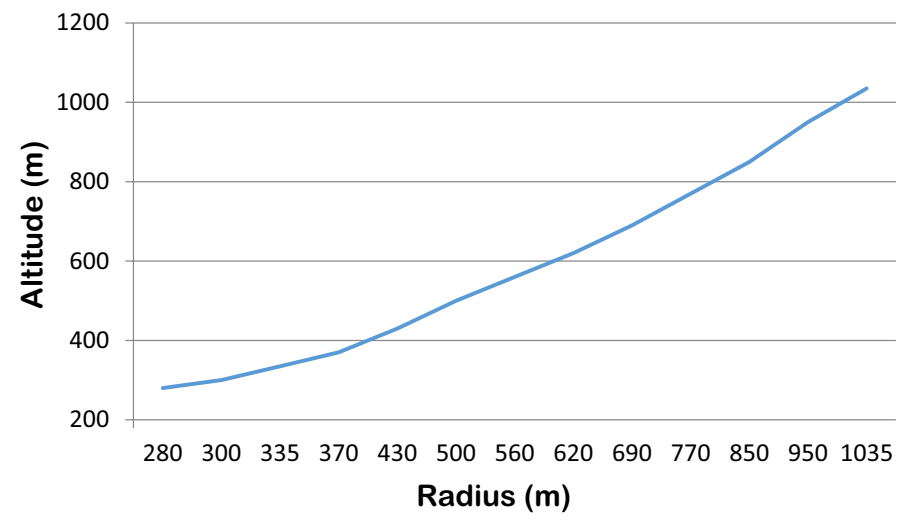

Fig. 6 Impact of UAV altitude on Tx power

airtime and limiting the research scope. Figure 6 shows the impact of UAV altitude on radius, and the radius tends to increase when the UAV increases its altitude, thus also affecting scattered users and the density that needs to be covered.

Increasing the altitude of a UAV also effects on its transmission power, and now the UAV requires more powerful transmission in order to cover the increased radius. The UAV are reliant on a limited size on chip battery. More power full transmission depletes the battery abruptly thus directly dropping the available amperes. Figure 7 

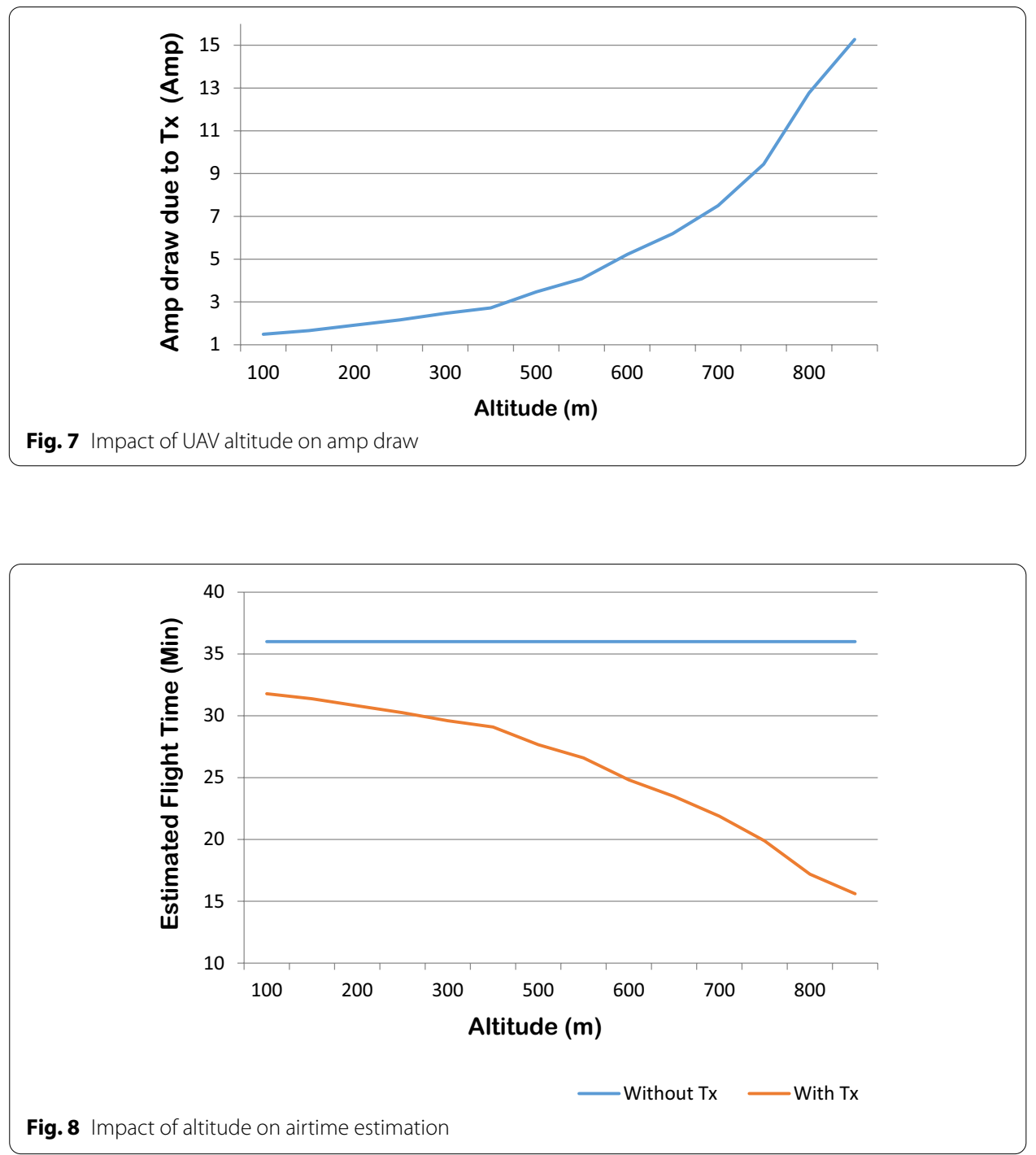

shows the same in comparison with the increasing altitude. The amp draw increases in linear fashion as the altitude is increased in start, but later on especially at altitude above $500 \mathrm{~m}$, the transmission power has a noticeable effect on the ampere draw of the battery and it results in less flight of a UAV. Thus, disrupting the service availability and suspension of the UAV operation.

Figure 8 shows the airtime estimation with and without considering the transmission impact in comparison with the altitude. It is witnessed that as the altitude is increased, the required transmission power also increases proportionally and it directly affects the estimated airtime of a UAV. It is worth to note that initially, the slope of airtime estimation decreases slightly but later on the decline on fast pace.

The difference discussed in Fig. 8 (with and without the transmission impact) is also calculated separately in order to access the scale of unavailability of UAV service as depicted in Fig. 9. This difference is substance depending upon the UAV application as the case may be of critical nature. 


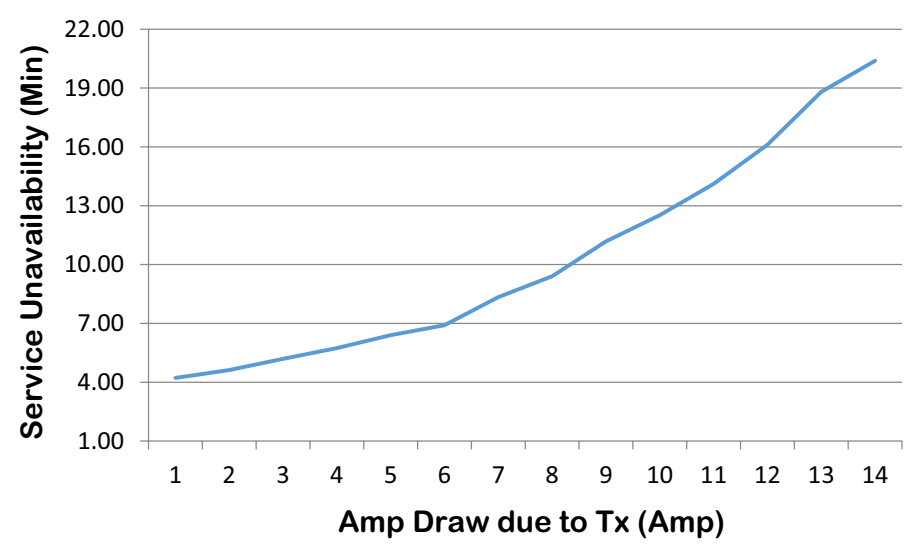

Fig. 9 Impact of amp draw on service availability

\section{Conclusion}

The UAVs deployment is largely dependent upon the available energy, and it is a key factor in UAV planning. The under- or overestimation of UAV air timing leads to wastage of resources or inefficiency of mission critical projects. Multiple factors influence the estimation of air timing but the majority of the literature concentrates on only flying time. In this research, the other factors are also accounted for that improved the estimation. In this research, the energy consumption is bifurcated into two usage scenarios (1) Implicit and (2) Explicit. The implicit usage was given its due weight in the previous solution; therefore, this research focused on explicit factors that have discussed in the literature but have not been given concentration in estimating the airtime and left as negligible factor. However, simulation has been performed and it is witnessed that the explicit factors of transmission power have severe effect on airtime estimation which is a factor not to be ignored in mission critical operation. In future, we will enhance the proposed idea and test with more parameters such as data centric fuzzy approach [37] and drone networking for marine applications [38].

\section{Abbreviations}

UAVs: Unmanned aerial vehicles; IoTs: Internet of things; COW: Cell on Wheel; BS: Base stations; UAV-BS: UAV base stations; DBS: Drone base station; PPMS: Photovoltaic power management system; EKF: Extended Kalman filter; PWR: Power required to carry one kilogram of weight; VOL: Battery voltage; BTS: Base transceiver station; GSM: Global System Mobile; UMTS: Universal Mobile Telephone Services.

\section{Authors' contributions}

SM has modeled and executed the research. AS and KNQ have supervised the research. SM has designed experimental testing and data visualization. AK and SI have validated mathematical design and testing. JL has enhanced the quality of the research by their valuable comments and suggestions in data analysis and discussion. SM has written the paper, where AS and KNQ, AK, SI and JL have improved the technical contents of the paper with comments and suggestions. All authors read and approved the final manuscript.

\section{Funding}

The research is funded by the Department of Computer Science, Iqra University, Islamabad Campus, Pakistan.

\section{Availability of data and materials}

The experimental data and associated settings will be made available to researchers and practitioners on individual requests with the restrictions that it will be used for further investigation with collaborative research progress only.

\section{Competing interests}

It is declared that there is no competing interest among authors. 


\begin{abstract}
Author details
${ }_{1}^{1}$ Department of Computing and Technology, I Ira University, Islamabad Campus, Pakistan. ${ }^{2}$ Department of Computer Science, Bahria University, Islamabad, Pakistan. ${ }^{3}$ School of Computing Science and Engineering (SCSE), Galgotias University, Greater Noida, Gautam Buddh Nagar, Uttar Pradesh 201308, India. ${ }^{4}$ University Institute of Information Technology, PMAS-AAUR, Rawalpindi, Pakistan. ${ }^{5}$ Universitat Politecnica de Valencia, 46022 Valencia, Spain.
\end{abstract}

Received: 30 September 2020 Accepted: 2 December 2020

Published online: 11 December 2020

\title{
References
}

1. K. Kumar, S. Kumar, O. Kaiwartya, A. Sikandar, R. Kharel, J.L. Mauri, Internet of unmanned aerial vehicles: QoS provisioning in aerial ad-hoc networks. Sensors 20(11), 3160 (2020)

2. M. Marchese, A. Moheddine, F. Patrone, IOT and UAV integration in $5 \mathrm{G}$ hybrid terrestrial-satellite networks. Sensors 19(17), 3704 (2019)

3. K. Kumar, S. Kumar, O. Kaiwartya, P.K. Kashyap, J. Lloret, H. Song, Drone assisted flying ad-hoc networks: mobility and service oriented modeling using neuro-fuzzy. Ad Hoc Netw. 102242 (2020)

4. X. Li, Deployment of drone base stations for cellular communication without apriori user distribution information, in 201837 th Chinese Control Conference (CCC) (IEEE, 2018), pp. 7274-7281

5. J.P. Pereira, I.M. Lopes, Next generation access networks: infrastructure sharing, in World Conference on Information Systems and Technologies (Springer, 2017), pp. 244-252

6. A. Mukherjee, N. Dey, N. Kausar, A.S. Ashour, R. Taiar, A.E. Hassanien, A disaster management specific mobility model for flying ad-hoc network, in Emergency and Disaster Management: Concepts, Methodologies, Tools, and Applications (IGl Global, 2019), pp. 279-311

7. K.N. Qureshi, A.H. Abdullah, O. Kaiwartya, S. lqbal, R.A. Butt, F. Bashir, A dynamic congestion control scheme for safety applications in vehicular ad hoc networks. Comput. Electr. Eng. 72, 774-788 (2018)

8. B. Perabathini, K. Tummuri, A. Agrawal, V.S. Varma, Efficient 3D placement of UAVs with QoS assurance in ad hoc wireless networks, in 201928 th International Conference on Computer Communication and Networks (ICCCN) (IEEE, 2019), pp. 1-6

9. J. Lu, S. Wan, X. Chen, P. Fan, Energy-efficient 3D UAV-BS placement versus mobile users' density and circuit power, in 2017 IEEE Globecom Workshops (GCWkshps) (IEEE, 2017), pp. 1-6

10. R. Yadav, W. Zhang, O. Kaiwartya, P.R. Singh, I.A. Elgendy, Y.C. Tian, Adaptive energy-aware algorithms for minimizing energy consumption and SLA violation in cloud computing. IEEE Access 6, 55923-55936 (2018)

11. C.-C. Lai, C.-T. Chen, L.-C. Wang, On-demand density-aware uav base station $3 d$ placement for arbitrarily distributed users with guaranteed data rates. IEEE Wirel. Commun. Lett. 8(3), 913-916 (2019)

12. S. Iqbal, K.N. Qureshi, N. Kanwal, G. Jeon, Collaborative energy efficient zone-based routing protocol for multihop Internet of Things, Transactions on Emerging Telecommunications Technologies (2020), p. e3885

13. F. Aadil, A. Raza, M. Khan, M. Maqsood, I. Mehmood, S. Rho, Energy aware cluster-based routing in flying ad-hoc networks. Sensors 18(5), 1413 (2018)

14. Y. Chen, D. Baek, A. Bocca, A. Macii, E. Macii, M. Poncino, A case for a battery-aware model of drone energy consumption, in 2018 IEEE International Telecommunications Energy Conference (INTELEC) (IEEE, 2018), pp. 1-8

15. A.A.A. Ateya, A. Muthanna, R. Kirichek, M. Hammoudeh, A. Koucheryavy, Energy-and latency-aware hybrid offloading algorithm for UAVs. IEEE Access 7, 37587-37600 (2019)

16. A. Fotouhi, H. Qiang, M. Ding, M. Hassan, L. G. Giordano, A. Garcia-Rodriguez, J. Yuan, Survey on uav cellular communications: practical aspects, standardization advancements, regulation, and security challenges, IEEE Communications Surveys \& Tutorials (2019)

17. L. Wang, B. Hu, S. Chen, Energy efficient placement of a drone base station for minimum required transmit power, IEEE Wireless Communications Letters (2018)

18. T.M. Cabreira, C. Di Franco, P.R. Ferreira, G.C. Buttazzo, Energy-aware spiral coverage path planning for uav photogrammetric applications. IEEE Robot. Automat. Lett. 3(4), 3662-3668 (2018)

19. M. Mozaffari, W. Saad, M. Bennis, M. Debbah, Efficient deployment of multiple unmanned aerial vehicles for optimal wireless coverage. IEEE Commun. Lett. 20(8), 1647-1650 (2016)

20. M. Mozaffari, W. Saad, M. Bennis, M. Debbah, Unmanned aerial vehicle with underlaid device-to-device communications: performance and tradeoffs. IEEE Trans. Wirel. Commun. 15(6), 3949-3963 (2016)

21. K. Li, W. Ni, X. Wang, R.P. Liu, S.S. Kanhere, S. Jha, Energy-efficient cooperative relaying for unmanned aerial vehicles. IEEE Trans. Mob. Comput. 15(6), 1377-1386 (2015)

22. A.E. Abdulla, Z.M. Fadlullah, H. Nishiyama, N. Kato, F. Ono, R. Miura, An optimal data collection technique for improved utility in UAS-aided networks, in IEEE INFOCOM 2014-IEEE Conference on Computer Communications (IEEE, 2014), pp. 736-744

23. L.D.P. Pugliese, F. Guerriero, D. Zorbas, T. Razafindralambo, Modelling the mobile target covering problem using flying drones. Optim. Lett. 10(5), 1021-1052 (2016)

24. H. Karl, An overview of energy-efficiency techniques for mobile communication systems, Report of AG Mobikom WG7 (2003)

25. L. Chiaraviglio, D. Ciullo, M. Meo, M.A. Marsan, I. Torino, Energy-aware UMTS access networks, ed: Citeseer (2008)

26. S. Jung, Y. Jo, Y.-J. Kim, Flight time estimation for continuous surveillance missions using a multirotor UAV. Energies 12(5), 867 (2019)

27. A. Fotouhi, H. Qiang, M. Ding, M. Hassan, L.G. Giordano, A. Garcia-Rodriguez, J. Yuan, Survey on UAV cellular communications: practical aspects, standardization advancements, regulation, and security challenges. IEEE Commun. Surv. Tutor. 21(4), 3417-3442 (2019) 
28. C. Lubritto, A. Petraglia, C. Vetromile, S. Curcuruto, M. Logorelli, G. Marsico, A. D'Onofrio, Energy and environmental aspects of mobile communication systems. Energy 36(2), 1109-1114 (2011)

29. R. Balani, Energy consumption analysis for bluetooth, wifi and cellular networks, Online Httpnesl Ee Ucla Edufwdocumentsreports2007PowerAnalysis Pdf (2007)

30. L. Zou, A. Javed, G.-M. Muntean, Smart mobile device power consumption measurement for video streaming in wireless environments: WiFi vs. LTE, in 2017 IEEE International Symposium on Broadband Multimedia Systems and Broadcasting (BMSB) (IEEE, 2017), pp. 1-6

31. M. Asif-Ur-Rahman, F. Afsana, M. Mahmud, M.S. Kaiser, M.R. Ahmed, O. Kaiwartya, A. James-Taylor, Toward a heterogeneous mist, fog, and cloud-based framework for the internet of healthcare things. IEEE Int. Things J. 6(3), 4049-4062 (2018)

32. F. Ullah, A.H. Abdullah, O. Kaiwartya, S. Kumar, M.M. Arshad, Medium access control (MAC) for wireless body area network (WBAN): superframe structure, multiple access technique, taxonomy, and challenges. Hum. Centric Comput. Inform. Sci. 7(1), 34 (2017)

33. A. Sawalmeh, N.S. Othman, H. Shakhatreh, Efficient deployment of multi-UAVs in massively crowded events. Sensors 18(11), 3640 (2018)

34. M.N. Ahmed, A.H. Abdullah, H. Chizari, O. Kaiwartya, F3TM: Flooding Factor based Trust Management Framework for secure data transmission in MANETs. J. King Saud Univ. Comput. Inform. Sci. 29(3), 269-280 (2017)

35. B. Haponiuk. Drone Flight Time Calculator. https://omnicalculator.com/other/drone-flight-time

36. K.N. Qureshi, A.H. Abdullah, O. Kaiwartya, F. Ullah, S. labal, A. Altameem, Weighted link quality and forward progress coupled with modified RTS/CTS for beaconless packet forwarding protocol (B-PFP) in VANETs. Telecommun. Syst. 75(pages145-160), 2020 (2016)

37. M. Prasad, Y.T. Liu, D.L. Li, C.T. Lin, R.R. Shah, O.P. Kaiwartya, A new mechanism for data visualization with TSK-type preprocessed collaborative fuzzy rule based system. J. Artif. Intell. Soft Comput. Res. 7(1), 33-46 (2017)

38. A. Khasawneh, M.S.B.A. Latiff, O. Kaiwartya, H. Chizari, Next forwarding node selection in underwater wireless sensor networks (UWSNs): Techniques and challenges. Information 8(1), 3 (2017)

\section{Publisher's Note}

Springer Nature remains neutral with regard to jurisdictional claims in published maps and institutional affiliations.

\section{Submit your manuscript to a SpringerOpen ${ }^{\circ}$ journal and benefit from:}

- Convenient online submission

Rigorous peer review

- Open access: articles freely available online

- High visibility within the field

- Retaining the copyright to your article

Submit your next manuscript at $\mathbf{s p r i n g e r o p e n . c o m ~}$ 